https://doi.org/10.1007/s10694-020-01074-x

\title{
Development of a Field Deployable Firebrand Flux and Condition Measurement System
}

\author{
Simone Zen (1D*, Jan C. Thomas, Eric V. Mueller and Bhisham Dhurandher, \\ School of Engineering, University of Edinburgh, Edinburgh, UK \\ Michael Gallagher, USDA Forest Service, Northern Research Station, New \\ Lisbon, NJ 15401, USA \\ Nicholas Skowronski, USDA Forest Service, Northern Research Station, \\ Morgantown, WV 26505, USA \\ Rory M. Hadden, School of Engineering, University of Edinburgh, Edinburgh, \\ $U K$
}

Received: 30 April 2020/Accepted: 23 November 2020

\begin{abstract}
A new instrument to quantify firebrand dynamics during fires with particular focus on those associated with the Wildland-Urban Interface (WUI) has been developed. During WUI fires, firebrands can ignite spot fires, which can rapidly increase the rate of spread (ROS) of the fire, provide a mechanism by which the fire can pass over firebreaks and are the leading cause of structure ignitions. Despite this key role in driving wildfire dynamics and hazards, difficulties in collecting firebrands in the field and preserving their physical condition (e.g. dimensions and temperature) have limited the development of knowledge of firebrand dynamics. In this work we present a new, field-deployable diagnostic tool, an emberometer, designed to provide measurement of firebrand fluxes and information on both the geometry and the thermal conditions of firebrands immediately before deposition by combining a visual and infrared camera. A series of laboratory experiments were conducted to calibrate and validate the developed imaging techniques. The emberometer was then deployed in the field to explore firebrand fluxes and particle conditions for a range of fire intensities in natural pine forest environments. In addition to firebrand particle characterization, field observations with the emberometer enabled detailed time history of deposition (i.e. firebrand flux) relative to concurrent in situ fire behaviour observations. We highlight that deposition was characterised by intense, short duration "showers" that can be reasonably associated to spikes in the average fire line intensity. The results presented illustrate the potential use of an emberometer in studying firebrand and spot fire dynamics.
\end{abstract}

\footnotetext{
* Correspondence should be addressed to: Simone Zen, E-mail: szen@ed.ac.uk
} 


\section{Introduction}

Firebrands are recognized as a crucial component of wildland fire spread and represent a threat to values at risk. Surveys of Wildland Urban Interface (WUI) fire events have highlighted the dominant role that firebrands play in the ignition of structures [1] and the ignition of spot fires ahead of the flame front [2] (see Fig. 1).

In simple terms, the firebrand hazard is related to the potential of firebrands to ignite new fires. In general, there are two possible mechanisms by which firebrands can start a spot fire: (1) a single firebrand with properties (e.g. temperature and mass) such that it can directly ignite a substrate; (2) the accumulation of firebrands in a location resulting in sustained smouldering combustion leading to ignition of a substrate. In both cases, the hazard associated with firebrands is governed by the geometry and temperatures of individual firebrands and the rate at which they are deposited on a substrate material.

Despite the clear importance of these parameters, relatively little is known about the size and temperature distribution of firebrands generated in real fires, or their rate of deposition (the firebrand flux). This is a complex problem with firebrand characteristics likely to be affected by the fuel properties, fire behaviours and environmental conditions.

Koo et al. [3] review the role of firebrands in historical, large-scale fires and give an overview of the problem. Investigations of wildland fires have reported information on the characteristics of firebrands generated. The work conducted by Rissel and Ridenour [4] on the Bastrop Complex Fire, Texas, USA provided information on firebrand dimensions. Using the area of holes in a trampoline located near the burned area as a proxy for projected area they showed that more than $90 \%$ of the holes showed a size less than $0.5 \mathrm{~cm}^{2}$ and that $85 \%$ were less than $0.05 \mathrm{~cm}^{2}$. In addition, they related the fire behaviour with the number of firebrands collected at a location and estimated that hot firebrands travelled distances

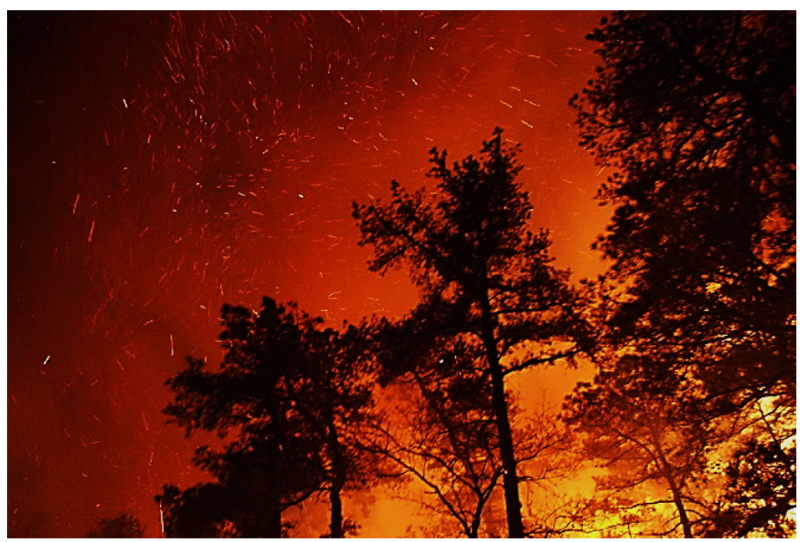

Figure 1. Example of firebrands generated during a wildfire. Prescribed fire, Franklin Parker Preserve, New Jersey Pine Barrens, USA, March 2017. 
of $30 \mathrm{~m}$ and note that cold firebrands were recorded up to 15 miles $(24 \mathrm{~km})$ from the fire.

The ultimate size and shape of a firebrand upon landing on a substrate will depend on, among other things, the distance that the firebrand travelled from the location it was generated. This will depend on many factors which are generally not known a priori (e.g. the local plume dynamics, the injection height, firebrand size). This issue has been explored by Manzello and Suzuki [5], who proposed using the Tachikawa number [6] (the ratio between aerodynamic and gravitational forces) as a proxy of the distance a firebrand can travel from the point it is generated. However, they did not find the expected relationship. This indicates that accurate prediction of the firebrand hazard requires knowledge of the flow field within the fire plume and the shape and temperature of firebrands. The complexity of this problem requires detailed field experiments which allow the firebrand characteristics and deposition dynamics to be related to fire behaviour and environmental variables.

Previous studies [7, 8] have relied on measurements of firebrands captured in prepared sites after the fire. This means that the temporal dynamics of firebrand deposition and the thermal, dimensional and geometric properties of the firebrands are not preserved. Furthermore, such techniques are labour intensive and not appropriate for capturing the firebrands generated from wildland fire events. However, methods with the specific purpose of collecting firebrands and extracting information pertaining to their generation [8], temporal flux, and geometry, and the associated fire behaviour [7] have been conducted. For example, Filkov et al. [9] recorded the number and temperature (from infrared thermography) of firebrands close to the ground and related this to physical measurements of hot firebrands. Data collected allowed a relationship between the number of falling firebrands and those flying through a control volume per second to be derived. A relationship between firebrand velocity and wind speed was also established. Results revealed that most of the firebrands are relatively cold $\left(<100^{\circ} \mathrm{C}\right)$ and their velocity is positively correlated to that of the wind while negatively correlated to their size.

Although approaches have been developed that aim to replicate firebrand exposure conditions experienced during a wildfire (e.g. [10-12]), there remains a need for field-based observations during actual wildland fire conditions, to ensure that the conditions explored in laboratory settings are representative of real scenarios.

In order to gather such data, we present a new apparatus which has been calibrated and tested to measure and analyse firebrand flux, size and temperatures that is field-deployable during field-scale fire events.

\section{The Firebrand Flux and Condition System}

Measurement

The firebrand flux and condition measurement system (emberometer) is composed of an aluminium rectangular body with a cross-section dimension of $250 \times$ $350 \mathrm{~mm}$. Firebrands are collected in an aluminium funnel (diameter of $550 \mathrm{~mm}$, 
located $400 \mathrm{~mm}$ above the body of the emberometer) and fall through a rectangular slot of $250 \times 100 \mathrm{~mm}$. As the samples enter the body of the emberometer, they pass in front of a back lit screen of $250 \times 350 \mathrm{~mm}$ (see Fig. 2).

Images of particles passing in front of this screen were captured by both a visible spectrum camera (Sony HD Handycam, framerate of $50 \mathrm{~Hz}$ ) and a longwave infrared (IR) camera, recording in the spectral range of 7.5-14 $\mu \mathrm{m}$ (FLIR A615). The emberometer length was designed to accommodate the IR camera at a distance which allowed the entire screen to be captured in a single frame. End caps are used to provide a controlled lighting environment.

Given the short residence time of the firebrands in front of the screen (on the order of $0.2 \mathrm{~s}$ ), a high frame rate is needed to ensure that each particle is captured in more than one frame (i.e. a minimum of $50 \mathrm{fps}$ ) thereby increasing the accuracy with which firebrand dimensions can be estimated. Furthermore, high shutter speeds are required to avoid a blurred image, and backlighting is used to record objects with high contrast and well-defined borders. Once particles have fallen in front of the screen, they are captured in a collection can to allow physical measurements for validation of the image analysis techniques.

Before proceeding with the analysis, it is worth discussing the use of the term 'flux'. In this work we are counting the number of falling firebrands that cross the area of the emberometer funnel. This area is known and therefore the flux could be expressed as the number of firebrands per unit area and time. However, accounting for the number of particles per unit area will possibly lead to an interpretation of the results as representative for the entire study area rather than at the measurement location. Therefore, in the following, we will refer to firebrand flux as the number of particles entering the emberometer per unit time.

Two distinct analyses were conducted: one for the video footage recorded from the visible (RGB) and IR camera positioned inside the emberometer; and one for a static image of the firebrands in the collection can. Figure 3 shows the schematic of the image analysis performed.

(a)

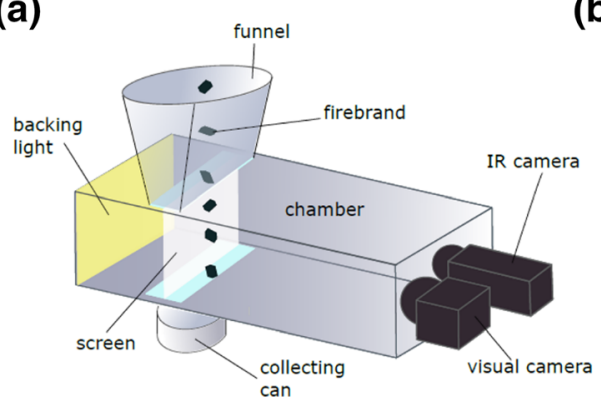

(b)

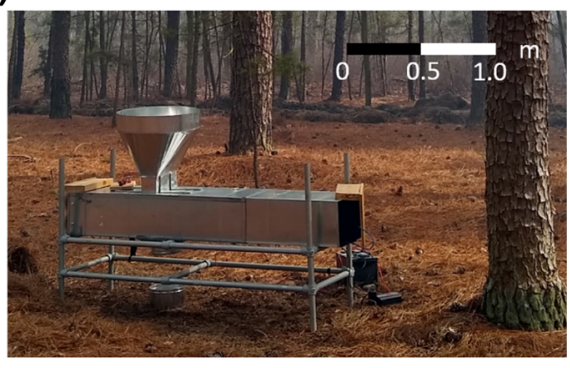

Figure 2. a Schematic of the emberometer structure and b the emberometer deployed in the field. 


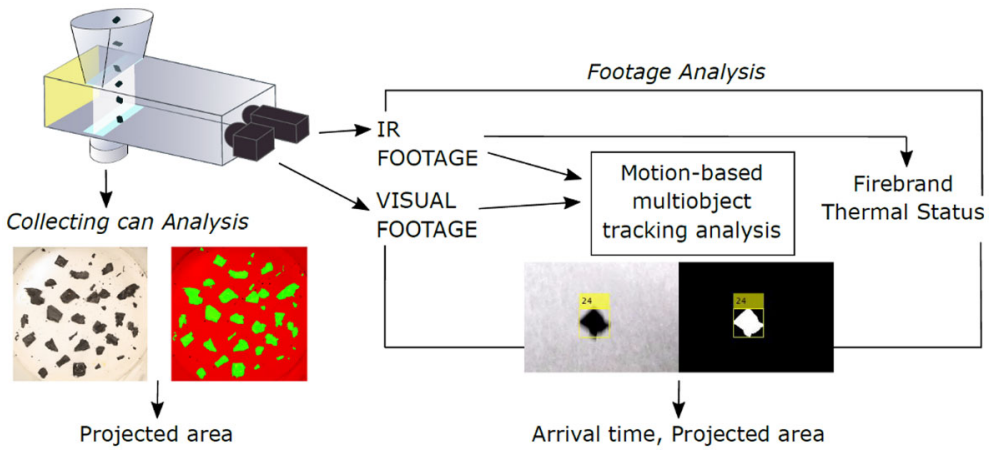

\section{Figure 3. The schematic of the workflow of the image analysis conducted. Image analysis techniques.}

\subsection{Analysis of the (Visible) RGB Footage}

To identify the number of firebrands and their properties (arrival time and dimensions), a motion based multi-object tracking technique was adopted [13]. This technique makes use of the free-falling nature of the particles to propagate information from frame to frame. For the analysis, the method identifies the background first and then extracts the foreground moving particles. In each frame, particles are identified, and a free-falling trajectory is computed for each of them, which allows the tracking of each particle from frame to frame. In the subsequent frame, the detected particles are assigned to the inferred trajectories to determine whether the object is the same particle identified in the previous frame or not. If no such match is found, a new tracking path is initiated, and the particle is counted as new firebrand. Therefore, for each object it is possible to know the centroid coordinate, the position in time and the projected area in each frame due to the simultaneous rotation ("tumbling") of the firebrand as it passes through the field of view. The steps of the method are detailed below:

(1) The images were corrected for inclination of the camera, frames were cropped to fit the backlit emberometer screen, and pixel size was calculated. In order to reduce processing time, frames which did not include any particles were filtered and removed. Frames were excluded from further analysis if they did not have any pixels in the blue band with digital numbers (DN) greater than 30 , which was the threshold at which particles saturated due to the high contrast of the backlighting. The frames in which firebrands were detected were then compiled into a new video so that additional image analysis could be performed.

(2) In order to account for variation in the background due to changes in light or instrument jitter, the background is defined by interpreting each pixel within the frame as a mixture of 3 normal Gaussian distributions, one for each colour band. Although the colour intensity of a pixel constituting the background is known, this will slightly change in time due to a noise given by the image recording, e.g. light variation. The mixture of Gaussians results in a 
multi-modal colour distribution for a pixel, which helps in recognizing foreground pixels in the presence of camera jitter [14]. This is required due to the camera jitter associated with the increase in (induced) wind intensity during the fire.

(3) Foreground particles are detected, and a series of morphological operations are applied. These operations modify the shape of an object according to the value of the neighbour pixels (i.e. erosion: remove pixels, dilation: add pixels). A further operation is conducted to cluster together pixels belonging to the same particle and generate the foreground 'blob' that represents the firebrand. This constitutes the track.

(4) Trajectories and tracks are estimated using a Kalman filter [15].

(5) In the following frame (or time step), the detected particles are assigned to a track by applying the Munkres' version of the Hungarian algorithm [16] to minimize the Euclidean distance between the centroid of the track and that of the detected object, i.e. cost. Once such a distance is computed, all the values are organized in a $m \times n$ cost matrix, where $m$ and $n$ are the number of tracks and detections, respectively. The maximum distance for the cost of not assigning a detected particle to a track was set equal to a 3-pixel square. This value was adjusted experimentally on the basis of particle size and velocity, and kept constant for all the analyses. If the distance threshold is too short, the model will separate the same track into multiple particles. Conversely, if the distance limit is too long, several particles may be included in one track.

(6) When a detection does not belong to any previous track, a new track is generated. When a particle has not been visible for a defined number of frames selected based on the problem under investigation, the track is deleted. This value was chosen to be three frames.

This process is applied at each pixel within a frame and for every frame. Computational time was reduced by preselecting the frames to be analysed based on the manual observation of firebrands. Each selected frame was time stamped to the original time of the experiment and each firebrand is labelled with a unique reference number according to the order of arrival from the ignition time. The result of the tracking analysis is a matrix containing the temporal values for centroid position and area of the firebrands. The arrival time was estimated as the time the firebrand enters the emberometer chamber (frame) for the first time. Before the start of an experiment, an object of known area was recorded on each camera to enable a re-scaling of size from, pixels to square-millimetres, for subsequently detected particles. This is a crucial step to compare dimensions of firebrands extracted from the footage with those extracted from the image of the collecting can.

\subsection{Analysis of the Infrared Footage}

The infrared footage was analysed following the framework presented above. This allowed the number of hot particles and their arrival time at the emberometer to be determined. 
For the field study, a threshold temperature of $100^{\circ} \mathrm{C}$ was used to discriminate between hot and cold particles. This threshold was identified during the calibration of the thermal camera in the laboratory and is discussed in Sect. 4.

A fixed range of temperature was selected from $5^{\circ} \mathrm{C}$ to $100^{\circ} \mathrm{C}$, and pixel colours extracted from the footage frames were scaled linearly, i.e. white and black corresponding to $5^{\circ} \mathrm{C}$ and $\geq 100^{\circ} \mathrm{C}$, respectively. Because true radiometric measurements of firebrand temperature are complicated by a number of factors, including the spectral emissivity [9], the temperatures associated with each pixel are not precise measurements. A range of emissivity values have been reported for wood and these can vary with factors such as surface temperature (including reacting state), moisture content, and spectral range considered. A value of 0.9 was used. The exact value is of less importance, as the infrared measurements are used to classify the state of the particles, guided by the laboratory calibration experiments, rather than to determine exact temperatures.

\subsection{Analysis of the Collection Can}

After passing through the image analysis, particles were collected for a posteriori analysis. Still images of the collection cans were taken to extract the number and projected area of the firebrands. The original RGB image was converted into a binary image, to differentiate the firebrands from the background. Morphological operations were applied to the binary images to estimate the shape of each firebrand within the collecting can. To allow conversion from pixels to area, a calibration picture was taken which included an object of known area. A US one-cent coin $(19.05 \mathrm{~mm}$ diameter $)$ and a $20 \times 20 \mathrm{~mm}$ square were used in the field and laboratory, respectively. The firebrands extracted from the collection can were used as ground truth for the validation of the analysis of the video footage. To do this, a minimum area was chosen, both for the analysis of the visual footage and the can image, below which pixel clusters recognized as firebrands were discarded. Overlapping or attached firebrands were carefully separated to avoid misinterpretation.

\section{Laboratory Calibration Experiments}

Laboratory experiments were conducted to assess the accuracy of the emberometer and image processing techniques using synthetically generated firebrands with known properties. Three types of calibration experiments were undertaken: evaluation of the particle size using cold particles; evaluation of the particle size and temperature using simulated cork firebrands; and evaluation of the particle size and temperature using synthetically generated bark firebrands.

\subsection{Particle Size Calibration}

First, simulated firebrands were cut from cork sheet (1-2 mm thick). The firebrands were cut into rectangles $12.5 \times 10 \mathrm{~mm}$ and $12.5 \times 20 \mathrm{~mm}$, such that their surface-to-volume ratio was $1.69 \mathrm{~mm}^{-1}$ and $1.59 \mathrm{~mm}^{-1}$, respectively. These sizes 
were representative of firebrands measured and reported in previous field studies [7, 8]. The cork pieces were painted black to provide higher contrast with the backlit screen and to represent a colour closer to that of charred organic material. A checkerboard image with $20 \times 20 \mathrm{~mm}$ squares was positioned in front of the camera to extract the dimensions of a single pixel and to allow the firebrand dimensions and area to be extracted from the images. For the lab experiments the pixel size ranges between $0.15 \mathrm{~mm}^{2}$ and $0.17 \mathrm{~mm}^{2}$. Change in pixel dimensions between experiments was due to different distance of the camera from the backlit screen. Hereinafter, we will refer to the firebrands with size $12.5 \times 10 \mathrm{~mm}$ and $12.5 \times 20 \mathrm{~mm}$ as small and large firebrands, respectively. The known initial geometry of the simulated firebrands was used as validation data to assess the image analysis performed on both the video (i.e. RGB and IR) and the static images of the collecting can. The area for each synthetic firebrand was estimated as the maximum projected area observed from the frames in which the firebrand was detected. The maximum temperature within each frame was extracted, and in the case of multiple particles (e.g. pieces detached from a reacting firebrand), this was associated to the firebrand with the largest area. Three trials were undertaken with small firebrands only and three with larger firebrands only. For each study, twenty firebrands were deposited into the emberometer funnel manually. Six studies were undertaken in which 40 synthetic firebrands (20 small, and 20 large) were deposited into the emberometer.

Figure $4 \mathrm{a}$ shows the area distribution of synthetic firebrands estimated from the image analysis for the cold experiments. Each area value is estimated as the maximum value of the projected areas observed for a single particle at different times. The projected area distribution of the larger firebrands is larger than the small firebrands. The central part of the distribution (i.e. 25th and 75 th percentile) of the estimated area for large firebrands ranges between $160 \mathrm{~mm}^{2}$ and $250 \mathrm{~mm}^{2}$, while for small firebrands it is comprised between $50 \mathrm{~mm}^{2}$ and $100 \mathrm{~mm}^{2}$. This wide range is due to the rotation of the particle while falling. Indeed, by rotating around three axes during its descent, small projected area values can be produced. This effect is apparent in Fig. 5 for both the particle sizes. The presence of areas above the maximum projected area expected of $250 \mathrm{~mm}^{2}$ in the distribution for large firebrands is due to overlapping with another firebrand or issues associated to motion blur and lighting, making it difficult to obtain well-defined boundaries of the firebrands.

The distribution of the projected area when measured using images of the collection can was much smaller compared to the emberometer (Fig. 4b). Area distributions for both small and large particles were quite narrow, with an overall variation of $23 \mathrm{~mm}^{2}$ and $45 \mathrm{~mm}^{2}$, respectively. The average estimated firebrand area for small particles increased from $105 \mathrm{~mm}^{2}$ to $128 \mathrm{~mm}^{2}$ (actual value 125 $\mathrm{mm}^{2}$ ) and from $205 \mathrm{~mm}^{2}$ to $240 \mathrm{~mm}^{2}$ (actual value of $250 \mathrm{~mm}^{2}$ ). This indicates that the visual analysis as implemented can reproduce the particle sizes (and that there were no changes to particle sizes as they landed in the collecting can) and that underprediction in the visual footage from the emberometer arises due to the issues previously discussed. 
(a)

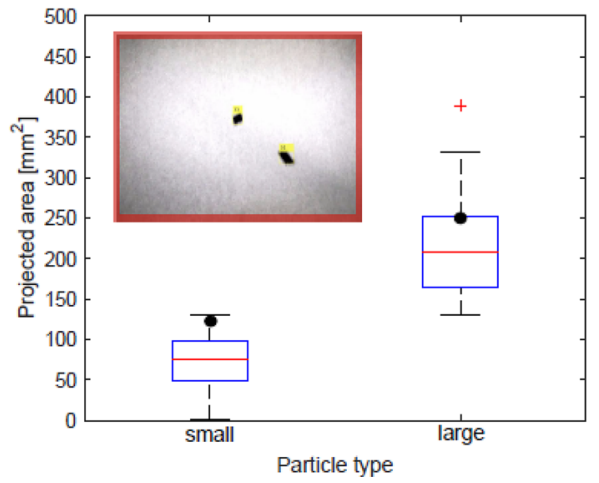

(b) collecting can

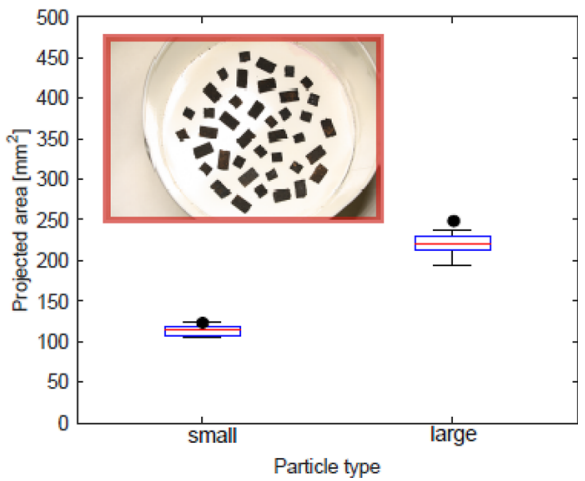

Figure 4. Boxplots for the projected area of the firebrands extracted from the a visual footage and b collecting can, in the laboratory experiments. The box indicates the portion of the distribution included between the 25th and 75 th percentile, the red line is the median of the distribution and the red cross the outliers. Black dots represent the actual area of the particles (Color figure online).
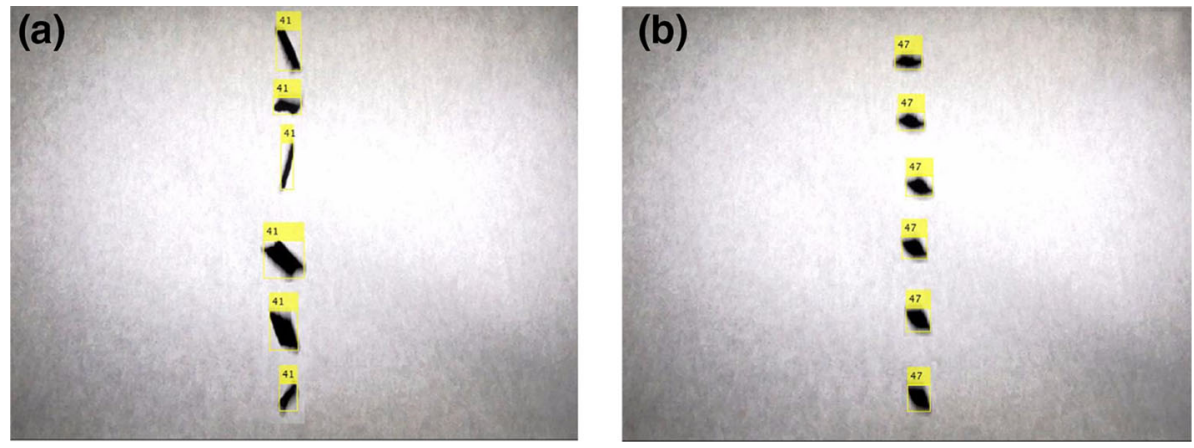

Figure 5. Frame montage showing the position of the falling particle at distinct times for both a large and $b$ small firebrands. The image shows how particle rotation induces large variations in the area recorded by the camera associated to large particles while it has minimal effect on the area associated to small particles.

\subsection{Particle Size and Temperature Calibration: Cork}

The hot experiments were performed to calibrate the infrared camera. Synthetic hot firebrands were created by heating cork particles in a muffle furnace at temperatures of $150^{\circ} \mathrm{C}, 250^{\circ} \mathrm{C}$ and $300^{\circ} \mathrm{C}$. Such temperatures were chosen to represent the ranges of different firebrand conditions: hot but not reacting firebrands $\left(150^{\circ} \mathrm{C}\right)$, charred firebrands $\left(250^{\circ} \mathrm{C}\right)$, and reacting firebrands $\left(300^{\circ} \mathrm{C}\right)$. Ten experiments divided between large and small firebrands were conducted to investigate whether a relationship exists between firebrand surface temperature and dimen- 
sions. It should be noted that in these experiments, the conditioning temperature did not result in significant shape changes of the cork.

Figure $6 \mathrm{a}$ shows the measured surface temperatures for particles heated to 150 , 250 and $300^{\circ} \mathrm{C}$. The results indicate that the surface temperature of non-reacting firebrands $\left(<300^{\circ} \mathrm{C}\right)$ dropped abruptly once removed from the muffle furnace. This is particularly evident from the results obtained for the firebrands heated up to $250^{\circ} \mathrm{C}$ that show temperatures in the range 70 to $170^{\circ} \mathrm{C}$ recorded using the IR camera. The data from Fig. $6 \mathrm{a}$ are used to define a threshold of $200^{\circ} \mathrm{C}$ to distinguish between reacting (hot) from non-reacting (cold) firebrands.

Particle size and temperature calibration (Fig. 6b) shows the distribution of the highest temperature recorded by the IR camera for small and large firebrands conditioned to $300^{\circ} \mathrm{C}$. The box represents the 25 th and 75 th percentile, the red line is the median value of the distribution and a cross indicates an outlier, i.e. values greater than $+/-2.7 \sigma$ from the mean of the distribution. The temperature distribution associated to large firebrands presents higher values, with almost the entire distribution falling above $300^{\circ} \mathrm{C}$. Small firebrands show a wider temperature distribution, and a lower median temperature of $230^{\circ} \mathrm{C}$ compared to that obtained for the large firebrands. Small firebrands have a slightly larger surface-to-volume ratio, which will increase the rate of cooling and hence the ability to sustain a combustion process.

The maximum temperature recorded in the IR camera and the associated particles are shown in Fig. 7. This demonstrates the ability to uniquely link each parti-

(a)

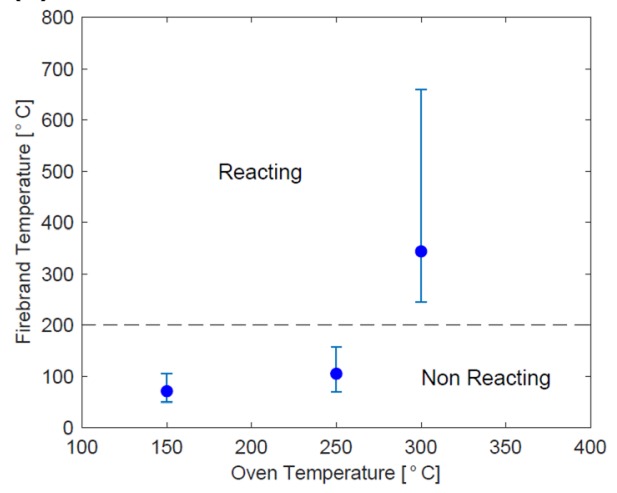

(b)

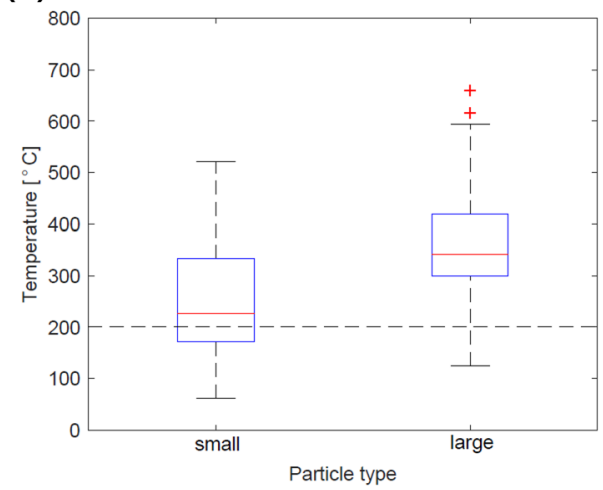

Figure 6. a Estimated temperatures recorded by the IR camera for the firebrands heated to temperatures of $150^{\circ} \mathrm{C}, 250^{\circ} \mathrm{C}$, and $300^{\circ} \mathrm{C}$. The marker indicates the average value while the bar represents the maximum and minimum value. b Boxplots for the temperature of small and large firebrands in the laboratory experiments. The box indicates the portion of the distribution included between the 25 th and 75 th percentile, the red line is the median of the distribution and the red cross the outliers, i.e. the values greater than $+/-2.7 \sigma$ from the mean (Color figure online). 
cle with a temperature measurement. Closer inspection allows further information to be obtained from the RGB images.

The presence of a red spot in firebrand 5 indicates that this firebrand is glowing, and this is consistent with the temperature measurement in excess of $500^{\circ} \mathrm{C}$. There is an appearance of a plume associated with firebrands 2 and 7, which again is indicative of a combustion process. Finally, firebrand 1 shows small pieces detaching from the firebrand, which indicates that the firebrand structure is changing. Although not explored further in this paper, these sources of information can be used to develop further insights into firebrand characteristics.

\subsection{Particle Size and Temperature Calibration: Bark}

Finally, a series of experiments was conducted using bark pieces. A total of 70 bark pieces of random size were conditioned in the muffle furnace. This allowed the physical measurements of firebrand dimensions before and after the experiments to be compared with those extracted from the visual footage and for the temperature recorded by the infrared camera to be related to firebrand conditions.

Having demonstrated the functionality of the system using idealised particles, further calibration was undertaken using synthetic firebrands generated from bark. Bark flakes ranging in size from $100 \mathrm{~mm}^{2}$ to $700 \mathrm{~mm}^{2}$ in size were mechanically removed from Pitch Pine (Pinus rigida Mill.) trees located in the New Jersey Pine
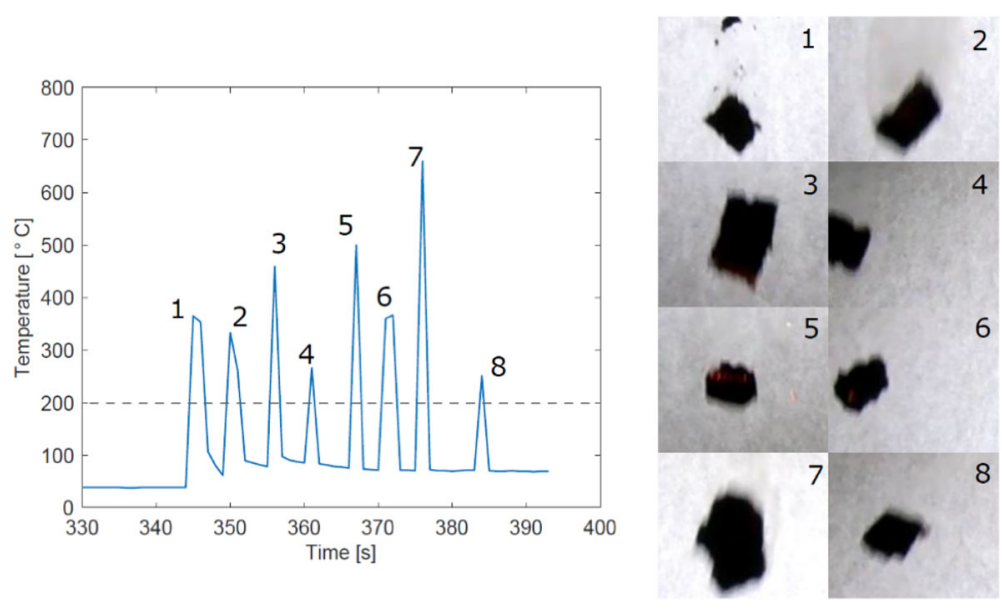

Figure 7. Example showing results from the combined use of the visual and IR camera. Maximum temperature recorded by the IR camera within 1 -s time window and images from the visual footage showing the related firebrands. The dashed line indicates the threshold discriminating between reacting (hot) and not reacting (cold) firebrands. 
Barrens, USA. This species was chosen to align with the vegetation used in the field deployment. [17]. The bark pieces were heated in the muffle furnace at $500^{\circ} \mathrm{C}$ before being dropped into the emberometer.

The cumulative distribution of the bark pieces is shown in Fig. 8a, and a typical selection of particles before conditioning is shown in Fig. 8b. A total of 70 bark pieces were used in this study, and approximately $80 \%$ of the pieces had an area between $100 \mathrm{~mm}^{2}$ and $400 \mathrm{~mm}^{2}$ prior to conditioning.

A total of 76 firebrands were detected by the image analysis (six more than were deposited into the emberometer) indicating the tendency of bark pieces to break up on heating. The detected firebrand sizes were less than those measured prior to heating and insertion into the emberometer, due to due to mass loss associated with particle heating chemical processes that continued while the particle travelled through the emberometer, and due to the previously mentioned underestimation that is common due to particle orientation in the view space. However, further analysis of the collection can indicates that the effect of the particle rotation is small relative to the consumption of the particle by burning. 123 firebrands were detected in the collection can and these were again shifted to smaller sizes than the image analysis, suggesting further fragmentation (assumed to be due to impact). A small percentage (tail of the distribution above the 90th percentile) of the firebrands collected in the can (dashed yellow line) are larger than the maximum size measured in the visual analysis (continuous red line). This may be due to the rotational effects described previously or the particles overlapping in the can. The total firebrand area from all particles in the visual analysis was 7,730 $\mathrm{mm}^{2}$ and the total firebrand area in the can analysis was $10,500 \mathrm{~mm}^{2}$.

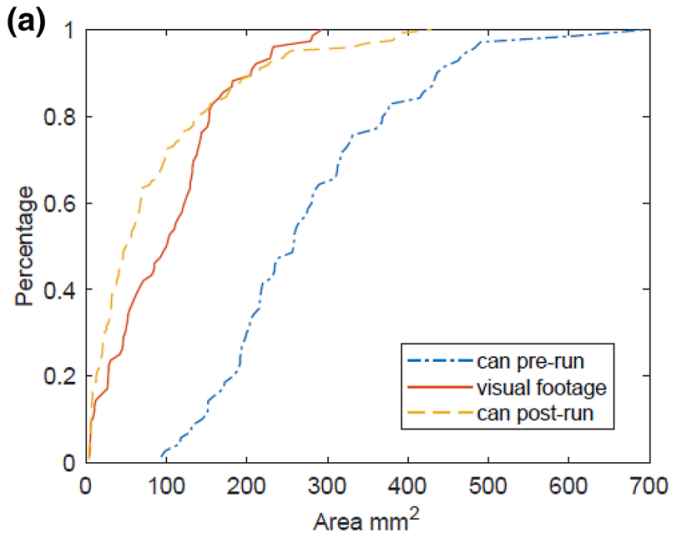

(b)

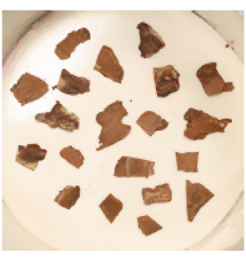

(c)

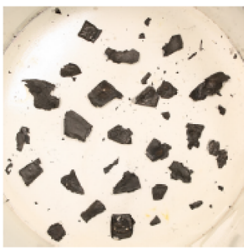

Figure 8. The graph a shows the cumulative distribution for the firebrands' projected area extracted from the image of the collecting can before (point-dashed line) and after (dashed line) the laboratory run, and from the visual footage (continuous line). The number of firebrands recorded from the can pre-run, the visual camera and the can post-run are, respectively, 70, 76, and 123 . Images show an example of collecting cans b before and a after the laboratory runs. 
Using infrared temperature measurements of the bark pieces, a temperature threshold for classification was developed. All particles were observed to be reacting upon deposition into the emberometer. The recorded infrared temperature range was between 20 and $570^{\circ} \mathrm{C}$. Particle temperature is plotted against particle size in Fig. 9. A weak positive correlation with particle size is observed. Based on these data, a temperature threshold of $100^{\circ} \mathrm{C}$ is proposed for use in field experiments to differentiate between hot and cold firebrands. This leads to $12 \%$ of the reacting particles being identified as cold.

The discrepancy in the temperature threshold between the cork and the bark indicates that this value is dependent on the firebrand geometry, material properties (e.g. emissivity, density) and burning characteristics (e.g. oxidation rate) of the materials. This means that the threshold value must be calibrated for a given system.

\section{Field Measurement of Firebrand Characteristics and Dynamies}

Two field-scale fire experiments were conducted, at the Franklin Parker Preserve in the New Jersey Pine Barrens, USA in March 2017 and March 2019. The New Jersey Pinelands is a high fire-frequency forest region dominated by highly fireadapted pines whose flakey-bark, for over a century, has been cited as a critical source of embers that contribute to the enhancement of wildfire spread $[9,18,19]$. Hereinafter we will refer to the 2017 experiment as Parker Preserve North (PPN) and the 2019 as Parker Preserve West (PPW). The study areas were each approximately 6.25 ha, with overstory vegetation mostly dominated by pitch pine (Pinus rigida Mill.) and tree oaks (Quercus spp.). The understory consisted of shrub oaks (Quercus spp.), blueberry (Vaccinium spp.), and huckleberry (Gaylussacia spp.).

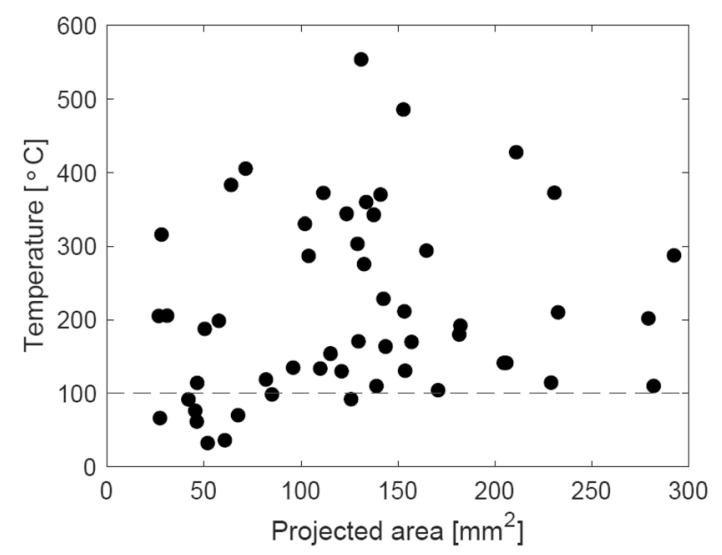

Figure 9. Relationship between firebrand projected area and temperature for the pieces of bark used in the laboratory hot runs (Color figure online). 
For a detailed description of the species assemblages of the New Jersey Pine Barrens $[20,21]$ and examples of experimental fire behaviour and firebrand production, see [8] and [22]. This section first discusses the measured fire behaviour to provide context for the firebrand measurements.

\subsection{Fire Behaviour}

The progression of the fire front in these experiments was tracked using an array of GPS-enabled single channel temperature measurement devices. These were deployed in a $10 \times 10$ array over the burn area, with a spacing of $25 \mathrm{~m}$. The localized fire arrival times, determined from the temperature signal, were interpolated to create isochrones of fire position. The inverse gradient of the fire arrival times was then used to obtain spread rate values. Figure 10 provides information on the spatial position of the fire front during the experiments at a time interval of $1 \mathrm{~min}$ and $2 \mathrm{~min}$, respectively for PPN and PPW. The shaded contour plot represents the local rate of spread computed from these fire front positions.

The average rate of fire spread in PPN was $0.26 \mathrm{~m} / \mathrm{s}$ compared to $0.09 \mathrm{~m} / \mathrm{s}$ for PPW. This can be partially explained by the dominant wind direction. In both cases the fire was ignited as a line on the northwest edge. Therefore, in the PPN experiment the wind pushed, on average, the fire along a direction perpendicular to the ignited edge. On the other hand, during the PPW experiment the wind shifted several times, yielding an average wind direction of $\sim 45^{\circ}$ to the ignited edge (Fig. 10).

Two emberometers were deployed for each experiment. In the PPN experiment, both emberometers were positioned outside of the burn, 25 and $50 \mathrm{~m}$ perpendicular to the edge of the burn unit (Fig. 10). During the PPW experiment one emberometer was deployed inside the burning plot and the other immediately outside at $25 \mathrm{~m}$ from the plot edge (Fig. 10). In the following, we will refer to the closest and further emberometer of the PPN burning as N1 and N2, respectively. Analogously, the emberometers inside and outside the plot in the PPW burning will be respectively named $\mathrm{W} 1$ and $\mathrm{W} 2$. W1 was located within the plot to evaluate very short-range firebrand deposition and was buried leaving only the collection funnel above the ground. After the calibration operation required to rescale the size from pixel to $\mathrm{mm}^{2}$, the size of a pixel for the images recorded from each emberometer deployed is: N1 $0.05350 \mathrm{~mm}^{2}$, N2 $0.06539 \mathrm{~mm}^{2}, \mathrm{~W} 10.14871 \mathrm{~mm}^{2}$, W2 $0.15805 \mathrm{~mm}^{2}$.

\subsection{Firebrand Characteristics}

The number and size distributions of firebrands collected at sites N1, N2 and W1 are shown in Fig. 11. Key information is summarised in Table 1. The data show that a large number of firebrands were collected during the experiment conducted at PPN compared to those collected at PPW. Hot firebrands were only observed at N1. The size distribution is consistent among sites N2, W1, and W2, but tends towards higher values for N1.

The distribution of the maximum projected area for all the firebrands collected during the field experiments are shown in Fig. 11 with distinction made between 

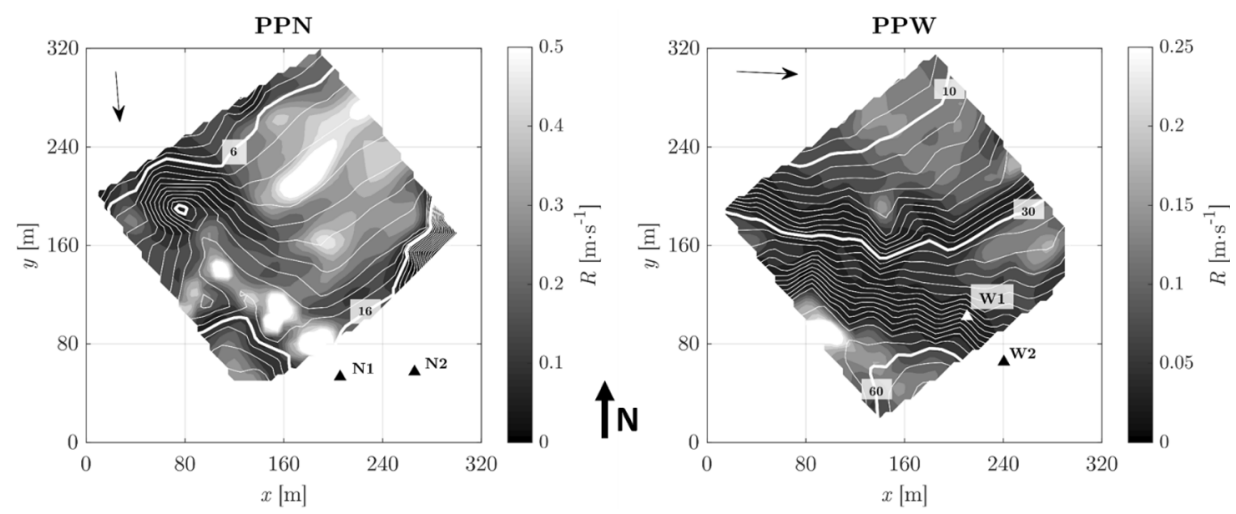

Figure 10. The study area. Representation of the two burning plots PPN and PPW, and the position of the two emberometers deployed in each experiment $N 1$, N2 and W1, W2, respectively. The shaded contour map shows the rate of spread computed from the position of the fire front recorded during the experiments and represented by the contour lines; the Iabels highlight some specific times from the ignition, for reference. The black arrow indicates the average wind direction.

hot (brown bars) and cold (blue bars) firebrands. As for the experiments using bark, data from the field does not show a clear relationship between firebrand size and surface temperature. Due to the very few firebrands collected at the site W2 this site does not present a significant distribution of the maximum projected area and thus it was omitted from the results.

The area distribution extracted from N1 shows $83 \%$ of the firebrands have an area $\leq 50 \mathrm{~mm}^{2}$ and $93 \% \leq 100 \mathrm{~mm}^{2}$. The firebrands collected at $\mathrm{N} 2$ and $\mathrm{W} 1$ are smaller with a maximum area around $70 \mathrm{~mm}^{2}$ and an average area of $31.5 \mathrm{~mm}^{2}$ (N2) and $13.8 \mathrm{~mm}^{2}$ (W1). Furthermore $93 \%$ of the collected firebrands show an area $<40 \mathrm{~mm}^{2}$ for the embrometers $\mathrm{N} 2$ and $\mathrm{W} 1$. These findings are in agreement with previous field observations $[4,7,8]$.

Comparisons of the area extracted from the visual footage to that from the static image of the can at the end of the field experiments are shown in Fig. 12. Qualitative agreement is observed between the curves for N1 and W1. In the case of $\mathrm{N} 2$, the curves overlap only for areas larger than $50 \mathrm{~mm}^{2}$ and the curves differ significantly for W2. However, in this latter case the few firebrands collected (only 7 ) and their small dimensions $\left(90 \%\right.$ of the firebrands below $15 \mathrm{~mm}^{2}$ ) diminish the significance of the distribution. The low number of firebrands collected at N2 (29) also limits the comparison between the two curves.

\subsection{Firebrand Dynamics}

The primary motivation behind the emberometer is to determine the time-dependent firebrand dynamics and to relate this to fire behaviour. Figure 13 shows the cumulative number of firebrands collected in the PPN and PPW fires alongside 
Fire Technology 2021

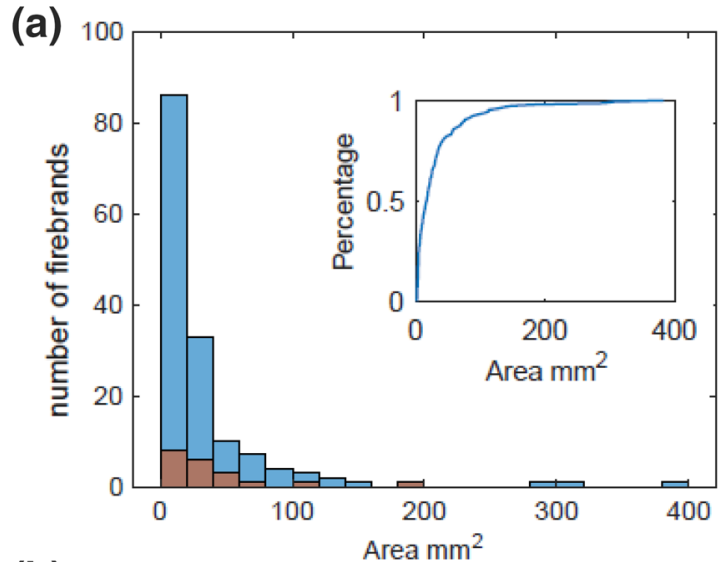

(b)

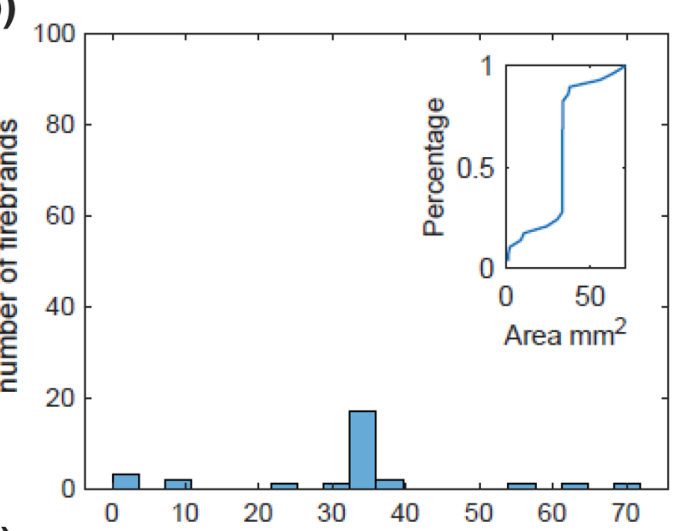

(c)

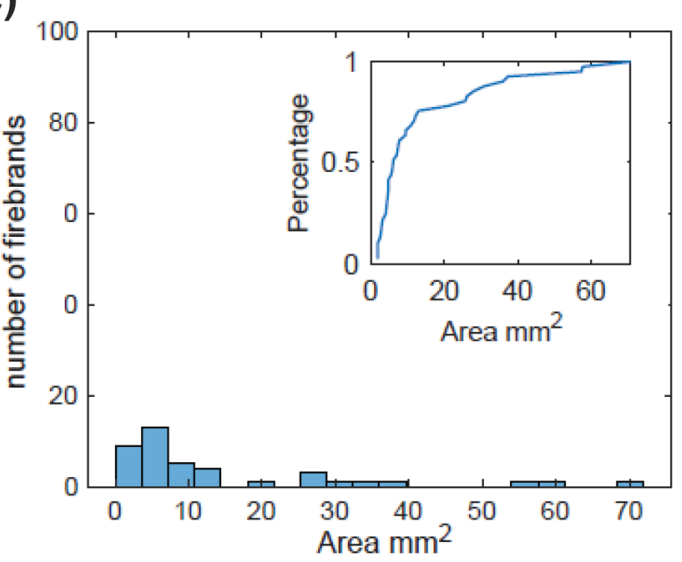


4Figure 11. Histogram and cumulative curve (inset) for the area of the firebrands collected at a N1, b N2, and c W1, as extracted from the analysis of the visual and thermal footage. The brown and blue bars are associated, respectively, to hot and cold firebrands. Due to the very few firebrands collected at the site $W 2$ this site does not present a significant distribution of the maximum projected area and thus it was omitted from the results (Color figure online).

Table 1

Characteristics of Firebrands Detected at Each Measurement Site

\begin{tabular}{lllc}
\hline Location & Total firebrands & Total hot firebrands & 10-90th percentile size range, $\mathrm{mm}^{2}$ \\
\hline N1 & 150 & 20 & $2.67-74.9$ \\
N2 & 29 & 0 & $2.07-37.8$ \\
W1 & 41 & 0 & $1.79-35.98$ \\
W2 & 7 & 0 & $2.3-24.0$ \\
\hline
\end{tabular}
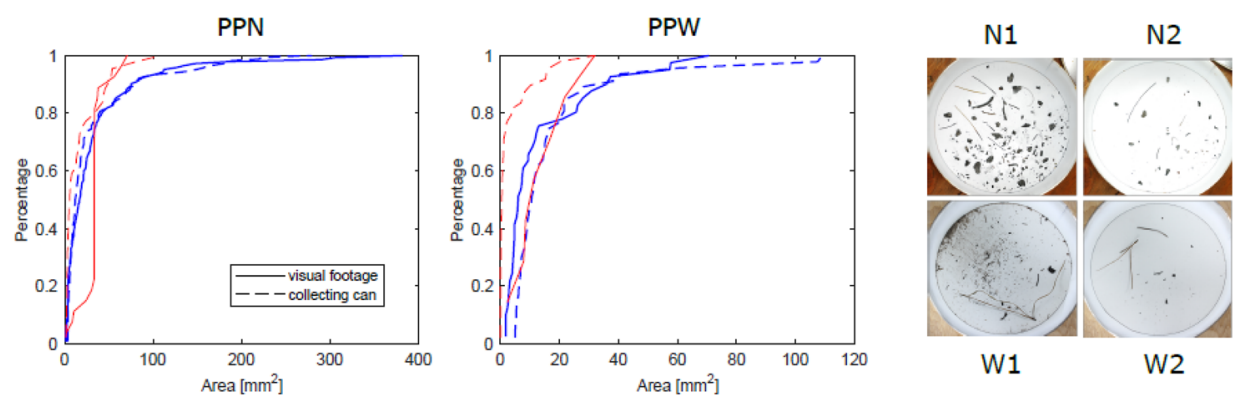

Figure 12. Cumulative distribution of the firebrands area extracted from the visual footage (continuous line) and the collecting can (dashed line) for the PPN and PPW burning. Line thickness and colour indicates the emberometer: the thick blue lines are associated to $\mathbf{N}$ and $W 1$ while the thin red lines to N2 and W2. The image shows the collecting can for each of the emberometers (Color figure online).

the estimated rate of spread. The average rate of spread, which can be correlated to the fire intensity, is approximately three times higher in the PPN compared to PPW. This demonstrates that higher fire intensities result in a larger number of firebrands (at approximately comparable locations and positions relative to the fire front).

Distinct periods of high deposition of firebrands occurred during each experiment, as illustrated by curve in Fig. 13 and Fig. 14. These firebrand showers are evident at location $\mathrm{N} 1$ between $12-14,15-17$, and 19-21 min from the fire ignition. At position N2, a smaller number of firebrands were recorded but again 

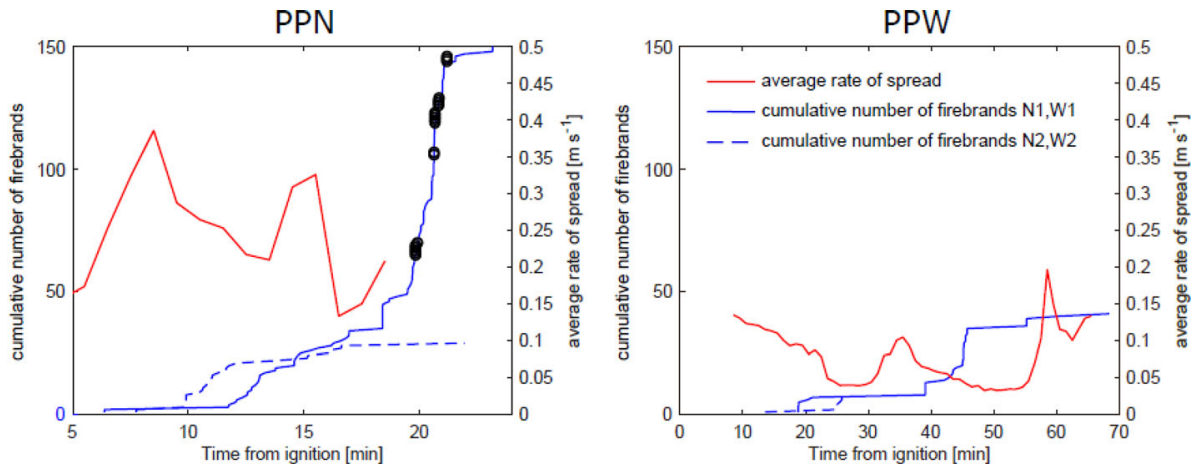

Figure 13. Temporal distribution of the firebrands for the emberometers deployed in the field (blue lines) and the average rate of spread estimated with a 1 min frequency (red line)-respectively emberometer $\mathbf{N} 1$,W 1 (blue continuous line) and emberometer N2,W2 (blue dashed line). The black circles indicate the presence of hot firebrands within the firebrand shower (Color figure online).

there is evidence of deposition occurring in two distinct showers between 10-12 and 15-16 min after ignition. The final shower at N1, resulting in hot particles, may be associated with the period of intense fire behaviour (high spread rate) identified immediately upwind of the N1 location (Fig. 10), which was less likely to impact N2. The number of firebrands collected during the PPW experiment was 41 and 7 at locations W1 and W2, respectively. For emberometer W1, it is possible to recognize two firebrand showers occurring approximately $19 \mathrm{~min}$ and 40 min after the ignition.

If it is assumed that the firebrands collected were generated during the periods of high spread rates, then the average travel time of a firebrand is approximately 5 min (see Fig. 13). This transit time is likely to be an upper estimate as there is some evidence to suggest that firebrands are generated after the passage of the fire front.

\section{Practical Considerations}

The above data show that coupled visual and IR image analysis can allow insights into the temporal nature of firebrand flux and allow relationships to be made between firebrand deposition and fire behaviours. The current technique however is constrained by several practical issues which are explored below.

\subsection{Measurement Locations}

The results presented above indicate that there is a large spatial variability in firebrand deposition due to factors such as fire behaviour, and plume dynamics. This is captured in Fig. 15, which shows the firebrand density measured using an array of collection cans in the PPW fire. The results indicate that the maximum fire- 
brand density was as high as $300 \mathrm{pcs} \mathrm{m}^{-2}$ adjacent to areas of rapid fire spread (and hence high fire intensity).

Firebrands were collected in discrete firebrand collection sites each covering an area of $1.5 \times 3 \mathrm{~m}$ in which 15 collection cans were positioned. Details on the method used to generate the results reported in Fig. 15 are in the supplementary material attached to the paper.

Clearly this indicates the value of multiple devices covering a larger area in order to fully characterise the temporal and spatial firebrand distribution. To achieve this in practical terms, it is necessary to reduce the dimensions and cost of the emberometer.

\subsection{Reduction of Scale and Cost}

Optimising the emberometer for field deployment (small, lightweight, low cost) and obtaining high quality images for analysis (larger capture area, higher resolution imagery, etc.) requires a series of compromises to be made. Consumer-grade visual cameras have been shown to be adequate for obtaining data at sufficient quality to obtain reliable measurements for image processing. Indeed, the majority of the measurement uncertainty here is due to the tumbling nature of the particle as it falls. However, further laboratory and field testing would enable a firmer statistical understanding of actual and detected particle size.

The primary optimisation to reduce cost and scale would be to substitute the infrared camera with a modified consumer grade camera. Since it has been demonstrated that accurate measurement of a falling, rotating particle's temperature is complicated by the variability in orientation and actual temperature and the relatively low time in the field of view, a bespoke calibration is likely to be required so high resolution optical pyrometry is not required and alternative techniques may be appropriate [23]. Reducing the thermal information to this level would allow for significant cost reduction while only introducing the need for a laboratory calibration to determine the appropriate thresholds to distinguish between hot and cold firebrands.

\subsection{Some Uncertainty about Particle Recognition}

While the emberometer was shown to give a good estimate of overall particle size distributions when deployed in the field, a limit was found in terms of characterising fine particles such as pine needles. The large aspect ratio of such particles means that they are difficult to identify and are susceptible to variabilities in the lighting in the frame. This may result in misclassification as one particle is classified as multiple separate firebrands. Improvements to the image processing techniques e.g. creation of a shape library or tuning to expected firebrand material may be a useful development.

\subsection{Variable Fire Dynamics and Variable Distance to Flaming Front}

The spatial scale of the fire also presents a challenge when selecting the number and locations of sampling points. This is due to the limited information collected 
Fire Technology 2021

(a)

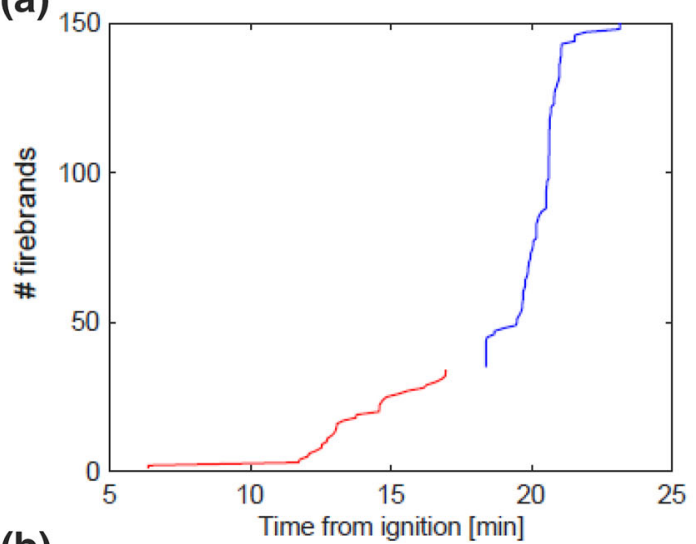

(b)
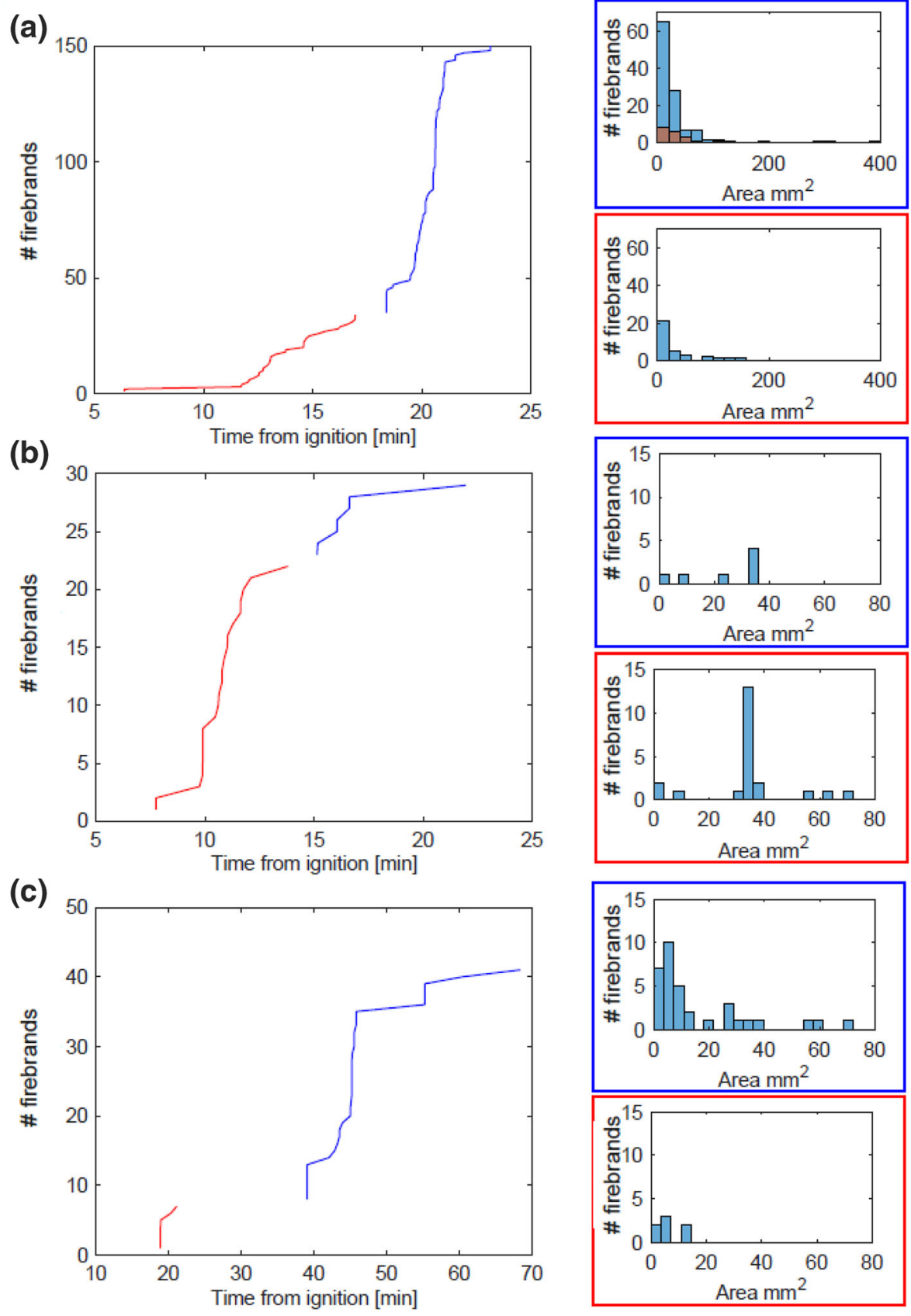
4Figure 14. Firebrand arrival time within the observed firebrand showers (distinct colours) for the emberometer a N1, b N2, and c W1. The coloured boxes are associated to the firebrand showers and indicate the firebrand distribution, according to their area. Due to the very few firebrands collected at the site W2, this site does not present a significant distribution of the maximum projected area and thus it was omitted from the results (Color figure online).

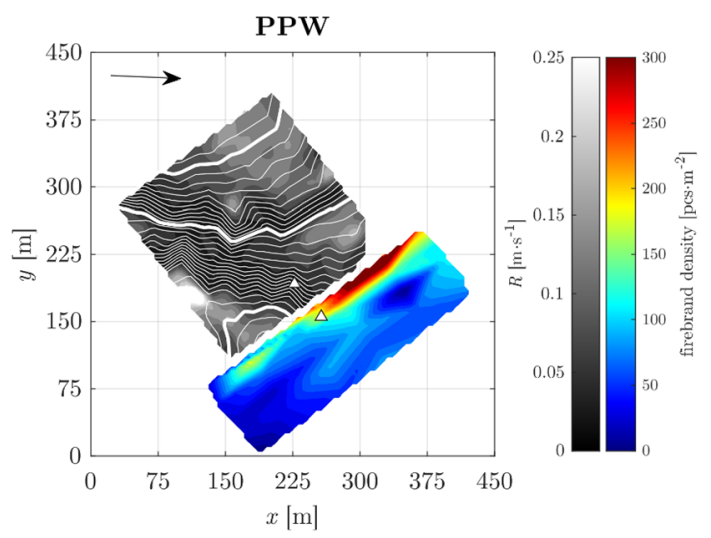

Figure 15. Contour plot obtained by interpolating the number of firebrands collected in each collection site during the PPW burning. The black and white image shows the average rate of spread of the fire at different points in time. The arrow on the top left of the graph represents the average wind speed and direction computed from the measurements taken during the field experiment. The triangles indicate the location of the two emberometers W1, and W2. The complete animation from which this image has been extracted can be found in the supplementary materials.

so far on the dynamics of firebrands generated during wildfires. In addition, changes in wind direction induce spatial variations in ROS that make it difficult to relate firebrand fluxes to the observed fire behaviour.

Subsequent information collected by deploying several emberometers during prescribed fires will relax such a simplification of fire dynamics and allow to spatially link fire dynamics with firebrand fluxes. Figure 15 shows an example of results obtainable. However, this is a static image presenting the distribution of deposited firebrands observed after the fire and does not account for the delay in the generation of firebrands, neither for the changes in wind direction. Therefore, in the supplementary material an animation has been added to show how time-resolved results can allow a firebrand deposition pattern to be associated to time changes in fire intensity and wind direction. 
These new relationships will further help future research to place the emberometers also during natural, i.e. uncontrolled, fires. Further information is also required on plume characteristics to allow the travel time of firebrands to be assessed. This would allow the firebrand fluxes (arrival times) to be strongly correlated to fire behaviours.

\section{Conclusions}

A tool for the monitoring of firebrand fluxes, sizes and thermal status has been developed, calibrated in the laboratory and deployed in field experiments. The analysis conducted has demonstrated the ability of the measurement and analysis techniques to provide new types of data on temporal firebrand dynamics. This method, if widely deployed, will rapidly allow a large coherent set of data to be generated to better understand the fire spread and structural ignition hazards arising from firebrands.

Using a combination of visual and infrared data, the emberometer overcomes the main issues associated with the collection and characterisation of firebrands. In particular, this new tool provides crucial new insights into firebrand dynamics by gathering reliable information on.

- the total number of firebrands and their real dimensions;

- the total volume and mass by combining the above information with vegetation type;

- the arrival time; and

- the thermal status of the firebrand approaching the ground.

Laboratory calibrations demonstrated the capability of the system to quantify primary firebrand characteristics in a controlled setting. It was found that the maximum projected area of the falling firebrand images gives a reasonable estimate of true firebrand size. Temperature thresholds could also be developed to differentiate between hot and cold firebrands. These thresholds were found to be material dependent.

In field experiments, the system was shown to be able to capture firebrand geometrical properties as well as discriminate between hot and cold firebrands. For the fires studied $\sim 9 \%$ of the particles were hot. No correlation between particle size and temperature was observed.

Currently the instrument is limited to research applications due to the size and cost. However, improvements have already been identified which would reduce the size and cost, thus increasing the ease of deployment.

By generating a coherent and consistent dataset through a common approach to measurement of the temporal dynamics of firebrands (and linking these to the fire behaviour) it is envisioned that significant advances in quantifying the hazards arising from firebrands can be made. 


\section{Acknowledgements}

We are grateful for the support of the New Jersey Forest Fire Service and the New Jersey Conservation Foundation in facilitating and managing the fires. We are thankful to the the National Institute of Standards and Technology (70NANB16H280); the Joint Fire Science Program (15-1-04-55); and the Strategic Environmental Research and Development Program (RC-2641) for funding which enabled this work. We also thank the three anonymous reviewers for their positive comments and suggestions that helped to further improve the manuscript.

\section{Open Access}

This article is licensed under a Creative Commons Attribution 4.0 International License, which permits use, sharing, adaptation, distribution and reproduction in any medium or format, as long as you give appropriate credit to the original author(s) and the source, provide a link to the Creative Commons licence, and indicate if changes were made. The images or other third party material in this article are included in the article's Creative Commons licence, unless indicated otherwise in a credit line to the material. If material is not included in the article's Creative Commons licence and your intended use is not permitted by statutory regulation or exceeds the permitted use, you will need to obtain permission directly from the copyright holder. To view a copy of this licence, visit http://creat ivecommons.org/licenses/by/4.0/.

\section{ELECTRONIC SUPPLEMENTARY MATERIAL}

The online version of this article (https://doi.org/10.1007/s10694-020-01074-x) contains supplementary material, which is available to authorized users.

\section{References}

1. Maranghides A, Mell W (2011) A case study of a community affected by the witch and guejito wildland fires. Fire Technol 47(2):379-420

2. Fernandez-Pello AC (2017) Wildland fire spot ignition by sparks and firebrands. Fire Saf J 91:2-10

3. Koo E et al (2010) Firebrands and spotting ignition in large-scale fires. Int $\mathbf{J}$ Wildl Fire 19(7):818-843

4. Rissel S, Ridenour K (2013) Ember production during the bastrop complex fire. Fire Manag. 72(4):7-13

5. Manzello SL, Suzuki S (2013) Experimentally simulating wind driven firebrand showers in wildland-urban interface (WUI) fires: overview of the NIST firebrand generator (NIST Dragon) technology. Procedia Eng 62:91-102

6. Holmes JD, Baker CJ, Tamura Y (2006) Tachikawa number: a proposal. J Wind Eng Ind Aerodyn 94(1):41-47 
7. Thomas JC et al (2017) Investigation of firebrand generation from an experimental fire: Development of a reliable data collection methodology. Fire Saf J 91:864-871

8. El Houssami $\mathrm{M}$ et al (2015) Experimental procedures characterising firebrand generation in wildland fires. Fire Technol 52:1-21

9. Filkov A et al (2017) Investigation of firebrand production during prescribed fires conducted in a pine forest. Proc Combust Inst 36(2):3263-3270

10. Manzello SL et al (2008) On the development and characterization of a firebrand generator. Fire Saf J 43(4):258-268

11. Fernandez-Pello AC et al (2015) Spot fire ignition of natural fuel beds by hot metal particles, embers, and sparks. Combust Sci Technol 187:1-2

12. Thomas JC, Mueller EV, Hadden RM (2017) 2018 "Estimating net heat flux from surrogate firebrand accumulations using an inverse heat transfer approach". Adv For Fire Res 2010:769-779

13. C. Stauffer and W. E. L. Grimson, 1999 Adaptive background mixture models for realtime tracking, in Proceedings. 1999 IEEE Computer Society Conference on Computer Vision and Pattern Recognition (Cat. No PR00149) 2 246-252

14. Magee DR (2004) Tracking multiple vehicles using foreground, background and motion models. Image Vis Comput 22(2):143-155

15. C. Ridder, O. Munkelt, and H. Kirchner, 1995 Adaptive background estimation and foreground detection using kalman-filtering," in Proceedings of the Recent Advances in Mechatronics, 193-199

16. Munkres TJ (1957) Algorithms for the assignment and transportation. Probl J Soc Ind Appl Math 5(1):32-38

17. El Houssami M, Lamorlette A, Morvan D, Hadden RM, Simeoni A (2018) Framework for submodel improvement in wildfire modeling. Combust Flame 190(12):24

18. Pinchot Gifford (1899) The relation of forests and fires. National Geographic 10:29

19. Pokswinski Scott, Gallagher Michael R, Skowronski Nicholas S, Louise Loudermilk E, O'Brien Joseph J, Kevin Hiers J (2020) Diurnal pine bark structure dynamics affect properties relevant to firebrand generation. Fire 3(4):55

20. Skowronski NS, Gallagher MR, Warner TA (2020) Decomposing the interactions between fire severity and canopy fuel structure using multi-temporal, active, and passive remote sensing approaches. Fire 3(1):7

21. Gallagher MR, Skowronski NS, Lathrop RG, McWilliams T, Green EJ (2020) An improved approach for selecting and validating burn severity indices in forested landscapes. Can J Remote Sens 46(1):100-111

22. Mueller EV et al (2017) Utilization of remote sensing techniques for the quantification of fire behavior in two pine stands. Fire Saf J 91(845):854

23. Urban JL, Vicariotto M, Dunn-Rankin D, Fernandez-Pello AC (2019) Temperature measurement of glowing embers with color pyrometry. Fire Technol 55(3):1013-1026

Publisher's Note Springer Nature remains neutral with regard to jurisdictional claims in published maps and institutional affiliations. 